\title{
EXPERIMENTAL EVOLUTION OF A PHEROMONE SIGNAL
}

3 Short title: Experimental sex pheromone evolution

5 Thomas Blankers ${ }^{1,2 \dagger}$, Elise Fruitet $^{1 \dagger}$, Emily Burdfield-Steel ${ }^{1 \dagger}$, Astrid T. Groot ${ }^{1}$

$6{ }^{1}$ Evolutionary and Population Biology, Institute for Biodiversity and Ecosystem Dynamics,

7 University of Amsterdam

$8 \quad 2$ Author for correspondence: thomasblankers< <at $>$ gmail.com

$9 \dagger$ These authors contributed equally

\section{SUMMARY STATEMENT}

12 This study reveals a mechanism that can facilitate evolution of sex pheromone components under

13 selection independent of other components of the sex pheromone that are not under selection.

\section{ABSTRACT}

16 Sexual signals are important in speciation, but understanding their evolution is complex as these

17 signals are often composed of multiple, genetically interdependent components. To understand

18 how signals evolve, we thus need to consider selection responses in multiple components and

19 account for the genetic correlations among components. One intriguing possibility is that

20 selection changes genetic correlations between components under selection and those not under

21 selection, reducing evolutionary constraints. However, this hypothesis remains largely untested

22 empirically. In this study, we investigate the evolutionary response of the multi-component

23 female sex pheromone blend of the moth Heliothis subflexa to 10 generations of artificial

24 selection. We observed a selection response of about $3 / 4$ s of a phenotypic standard deviation in

25 the components under selection. Interestingly, other pheromone components that are

26 biochemically and genetically linked to the components under selection did not change. We 
27 found that this component-specific selection response was likely facilitated due to reduced

28 genetic coupling between the component under selection and the components not under

29 selection, resulting from changes in genetic covariances within the 10 generations of selection.

30 Our findings provide rare empirical support for an intriguing mechanism by which a sexual

31 signal can respond to selection without possible constraints from indirect selection responses.

33 Key words: Sexual communication, Genetic variance-covariance, Pheromone, Artificial

34 selection, Lepidoptera

\section{INTRODUCTION}

37 Animal taxa that engage in sexual communication typically show high among-species diversity

38 in sexual signals (Andersson, 1994; Coyne and Orr, 2004; Ritchie, 2007; Schaefer and Ruxton,

39 2015; Wiens and Tuschhoff, 2020). Sexual signals are generally hypothesized to diversify due to

40 directional or disruptive selection (Ritchie, 2007; Schaefer and Ruxton, 2015; West-Eberhard,

41 2014; Wilkins et al., 2013), although their evolution is still a mystery in many species. Since

42 sexual signals play an important role in the origin and maintenance of species and contribute to

43 biodiversity (Coyne and Orr, 2004), it is important to assess whether there are constraints to their

44 selection response and identify the mechanisms that can mitigate those constraints.

45 Understanding selection responses in sexual signals is challenging, because signals are often

46 composed of multiple components (Candolin, 2003; Higham and Hebets, 2013; Rowe, 1999).

47 For example, mating songs can vary both in pitch and in rhythm (Blankers et al., 2015; Tanner et

48 al., 2017; Wilkins et al., 2015), color signals can be composed of multiple, functionally distinct

49 patches (Cole and Endler, 2015; Grether et al., 2004), and sex pheromones are often blends of

50 multiple chemical compounds (Ferveur, 2005; Linn et al., 1987). To understand how

51 multicomponent signals evolve, we thus need to consider the selection response in multiple

52 dimensions simultaneously. Signal components can have a shared genetic or developmental

53 basis, or can be subject to correlated selection pressures (Armbruster et al., 2014; Cheverud,

54 1996). The resulting genetic correlations among signals components can influence how selection

55 on the phenotype translates to changes in the underlying genotypes (Chenoweth and Blows, 
2006). To understand how the genotype-phenotype map of sexual signals influences the selection

response, we thus need to determine the genetic correlations between the different signal components.

Statistical frameworks in quantitative genetics, in particular the (multivariate) breeder's equation, allow us to predict and quantitatively understand selection responses in correlated traits. In this framework, the response to selection is a function of the genetic $(\mathrm{G})$ and phenotypic $(\mathrm{P})$ variance-covariance matrix and the selection gradient: selection acts on the P matrix and the resulting response is constrained by the G matrix (Lande, 1979; Lande and Arnold, 1983; Lynch and Walsh, 1998). The difficulty in predicting selection responses of multivariate traits is that selection acting on multiple components may be counterbalancing, e.g. directional selection on one trait, but stabilizing selection on correlated traits, resulting in evolutionary constraints (Barton and Turelli, 1989). Counterbalancing selection is likely prevalent in the evolution of sexual signals, as choosing individuals may favor higher or lower values of some component of the signal, while changes in correlated components may result in reduced mate attraction.

These evolutionary constraints can be overcome if genetic variances and covariances themselves respond to selection, thus reshaping the G matrix (Arnold et al., 2008; Barton and Turelli, 1989;

72 Eroukhmanoff, 2009; Jones et al., 2003; Melo and Marroig, 2014; Revell, 2007; Roff and

73 Fairbairn, 2012). Theory predicts that the G matrix will vary through time, because on one hand

74 selection erodes genetic variance (Barton and Turelli, 1989; Estes and Arnold, 2007), while on

75 the other hand mutation and introgression add new variation, albeit more slowly. Moreover, 76 genetic correlations can respond to selection directly, especially if they result from interactions 77 among unlinked genetic loci affecting the co-expression of multiple traits (Wolf et al., 2005), or

78 from selection acting on correlations directly (Armbruster et al., 2014; Roff and Fairbairn, 2012;

79 Svensson et al., 2021). Interestingly, directional selection can change genetic covariances and 80 increase modularity, meaning that groups (modules) of traits become genetically independent 81 from other modules, in a way that facilitates the phenotypic response to selection (Melo and 82 Marroig, 2014). Empirical work has shown that patterns of covariation can indeed evolve both 83 across populations and species in nature (Bégin and Roff, 2003; Berner et al., 2010; Blankers et 84 al., 2017; Garant et al., 2008; Gosden and Chenoweth, 2014) and during artificial selection 85 experiments (Careau et al., 2015; Hine et al., 2011; Uesugi et al., 2017). However, it is still 
unclear how changes in the phenotypic selection response are related to changes in genetic

87 (co)variance through time.

88 In this study, we explored the response in phenotypic means and genetic (co)variances to

89 artificial selection on the female sex pheromone of the moth Heliothis subflexa (Lepidoptera,

90 Noctuidae). Like many other moths, $H$. subflexa females secrete a sex pheromone blend to which

91 conspecific males are attracted. These sex pheromone blends are species-specific and vary

92 among species in both the presence/absence of components as well as in relative amounts (or

93 ratios) of the components (Cardé and Haynes, 2004; Schneider, 1992).

94 The sex pheromone blend of $H$. subflexa females consists of 11 compounds, with the following

95 components that are critically important for conspecific male attraction: (Z)-11-hexadecenal

96 (Z11-16:Ald) as the major sex pheromone component, (Z)-9-hexadecenal (Z9-16:Ald) and (Z)-

97 11-hexadecenol (Z11-16:OH) as the two secondary sex pheromone components, without which

98 H. subflexa males are not attracted (Groot et al., 2007; Vickers, 2002). Interestingly, the acetate

99 esters (Z)-7-hexadecenyl acetate (Z7-16:OAc), (Z)-9-hexadecenyl acetate (Z9-16:OAc), and (Z)-

100 11-hexadecenyl acetate (Z11-16:OAc), from here on referred to as "acetates", have a dual role:

101 these acetates attract conspecific males, while simultaneously repelling males of $H$. virescens

102 (Groot et al., 2006; Vickers and Baker, 1997). In geographic regions where H. virescens is

103 present, acetates are more abundant in the H. subflexa pheromone compared to where this

104 species is absent (Groot et al., 2009a). This suggests that the acetates are subject to divergent

105 selection across a geographic cline. The relative amounts of the other components are

106 hypothesized to be under stabilizing selection across the range, as in general in moth pheromone

107 communication the mean blend is preferred over deviations from the mean (Allison and Cardé,

108 2008; Groot et al., 2010; Kárpáti et al., 2013; Linn et al., 1997; Löfstedt, 1990; Zhu et al., 1997).

109 To explore the selection response of the acetates, we selected for higher and lower amounts of

110 acetates during 10 generations of truncation selection. Since the acetates vary geographically

111 (Groot et al., 2009a) and have a genetic basis that is partially independent of other components

112 (Groot et al., 2009b), we hypothesized that the relative amount of acetates can evolve in response

113 to univariate selection for higher/lower acetates but that genetic variance will be reduced after

114 selection. However, since acetates also partially share their genetic basis with other components

115 and since all sex pheromone compounds are produced through the same biosynthetic pathway, 
116 we also hypothesized that there will be indirect selection responses in other pheromone

117 components, specifically in the unsaturated aldehydes, Z9-16:Ald and Z11-16:Ald, and alcohol,

118 Z11-16:OH (Fig 1). Since some of the correlations among components may reduce the selection

119 response, we also expected genetic covariances to change during selection, specifically in a way

120 that facilitates the phenotypic selection response.

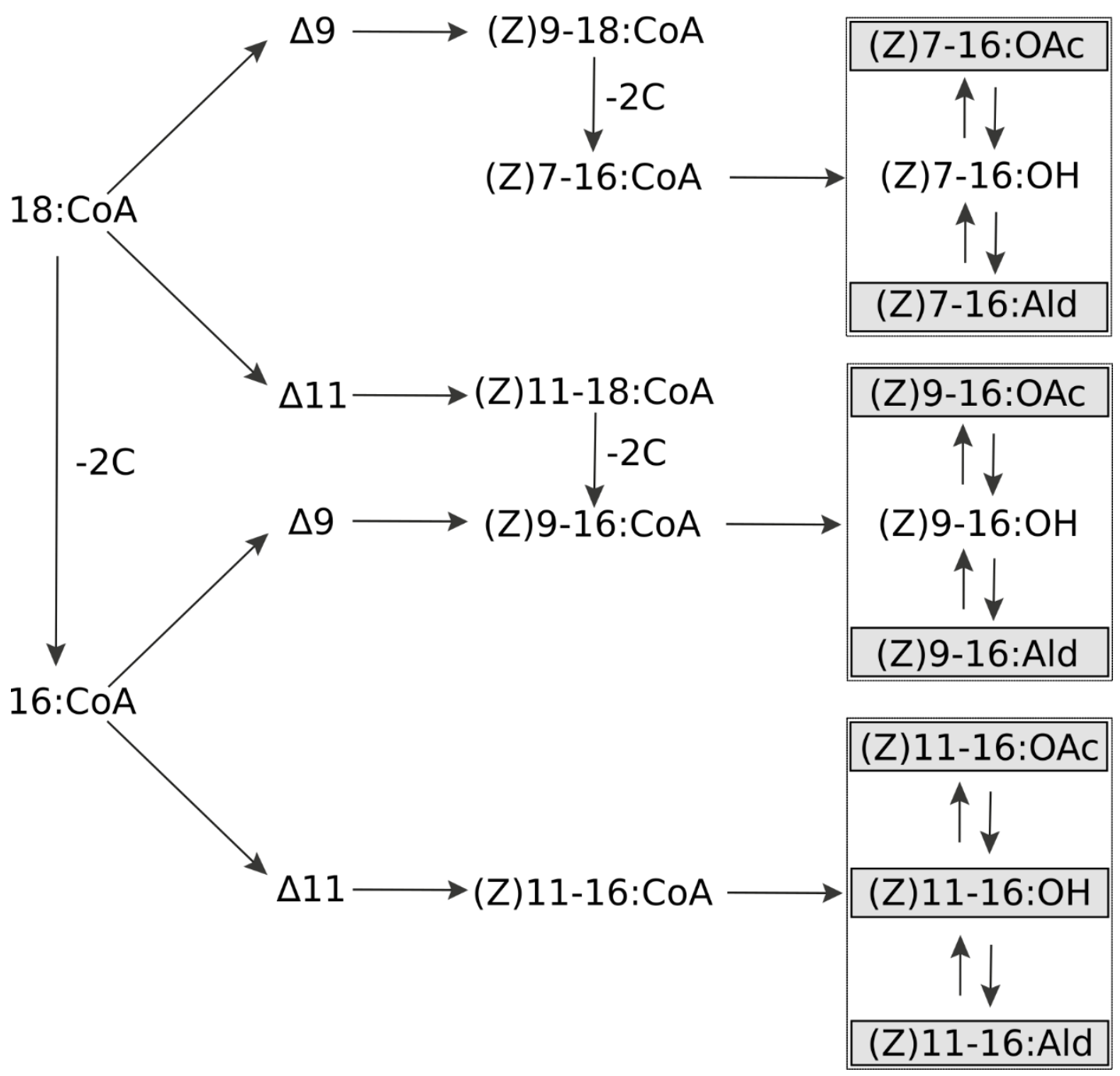

122 Figure 1. Simplified biosynthetic pathway of the Heliothis subflexa sex pheromone, modified from (Groot et al.,

$1232009 b$ ) with permission. Desaturation and $\beta$-oxidation produce mono-unsaturated acyl-CoA precursors from 18 or

12416 carbon acyl CoA derivatives, which are then modified to form acetate esters, alcohols, and aldehydes through

125 specific enzymatic conversions. The $\Delta 9$ and $\Delta 11$ desaturases create a double bond between the $9^{\text {th }}$ and $10^{\text {th }}$ or $11^{\text {th }}$

126 and $12^{\text {th }}$ carbon of the 18- or 16-carbon acyl-CoA derivatives, respectively. $\beta$-oxidation shortens the chain length

127 from 18 to 16 carbons. Note that (Z)9-16 acyl-CoA can be formed through two alternative routes. The compounds

128 present in the pheromone glands of $H$. subflexa are in grey boxes. 


\section{METHODS}

\section{Insect rearing}

132 The laboratory population of $H$. subflexa was founded from animals collected in the field in 2006

133 and has been reared at the University of Amsterdam since 2011 using single pair matings to

134 maintain genetic diversity. There has been occasional exchange among $H$. subflexa populations

135 at North Carolina State University, Amsterdam, and the Max Planck Institute for Chemical

136 Ecology in Jena. Eggs collected from single pair matings were kept in Petri dishes (Ø $85 \mathrm{~mm})$

137 with artificial wheat germ/soy flour-based diet (BioServ Inc., Newark, DE, USA) at room

138 temperature for approximately 10 days, after which larvae were reared in separate individual 37-

$139 \mathrm{~mL}$ cups filled with the same artificial diet and kept at $25^{\circ} \mathrm{C}$ and $60 \%$ relative humidity with

140 14h:10h light-dark cycle. Upon emergence, adults were provided sugar water. Pairs of males and

141 females were housed in $375 \mathrm{~mL}$ paper cups covered with gauze and kept under the same

142 conditions as the late-instar larvae and virgin adults. The mating pairs were provided with sugar

143 water. To stimulate oviposition, a freshly cut gooseberry fruit was placed on top of the gauze.

144 Once the eggs began to hatch, the gauze and the eggs and larvae on it, were transferred to Petri

145 dishes, which were then placed at room temperature until larvae were transferred to individual

146 cups. The females that produced fertile offspring were collected and phenotyped, if still alive.

147 All matings were assigned unique numbers. This way, we obtained a full pedigree of all

148 individuals in the selection and control lines.

\section{Phenotyping}

150 Female sex pheromone can be extracted from mated and old females by injecting them with

151 Pheromone Biosynthesis Activating Neuropeptide, i.e. PBAN (Groot et al., 2005). Females were

152 injected with a $7.5 \mathrm{pmol}(2 \mu \mathrm{L}$ of a $0.0146 \mu \mathrm{g} / \mu \mathrm{L})$ PBAN solution to activate pheromone

153 production in the mated females. After a 90-minute incubation time, female glands were

154 extruded by squeezing the abdomen then fixed by firmly holding the abdomen with forceps just

155 anterior of the gland. The gland was excised with microdissection scissors. Excess abdominal

156 tissue and eggs that remained in the ovipositor were removed, after which the glands were 
157 submerged in $50 \mu \mathrm{L}$ hexane containing $200 \mathrm{ng}$ pentadecane as an internal standard. After 30

158 minutes, the glands were removed and the extracts stored at $-20^{\circ} \mathrm{C}$ until analysis.

159 Pheromone extracts were analyzed by injecting the concentrated samples into a splitless inlet of a

160 7890A GC (Agilent Technologies, Santa Clara, CA, USA). The area under the pheromone peaks

161 was calculated using integration software implemented in Agilent ChemStation (version

162 B.04.03). Pheromone peak areas were obtained for the 11 pheromone components: two 14-C

163 aldehydes (14:Ald, Z7-14:Ald), four 16-C aldehydes (16:Ald, Z7-16:Ald, Z9-16:Ald, Z11-

164 16:Ald), the three acetates (Z7-16:OAc, Z9-16:OAc, Z11-16:OAc), and two 16-C alcohols (Z9-

165 16:OH, Z11-16:OH). Z7-16:Ald and Z9-16:Ald were difficult to separate by GC and were

166 therefore integrated as one peak (referred to Z9-16:Ald). Absolute amounts (in ng) of each

167 compound were calculated relative to a $200 \mathrm{ng}$ pentadecane internal standard. All downstream

168 analyses were done in R 3.6.1 (R Core Team, 2019). Samples containing < $20 \mathrm{ng}$ were excluded,

169 because the ratios of the components in such low titers cannot be reliably measured in the

170 chromatogram. Relative amounts were calculated by dividing the absolute amounts by the total

171 amount across all 11 components.

\section{Data transformation}

173 Selection was performed based on relative amounts of acetates. However, describing

174 relationships among relative amounts is problematic, because they sum to $100 \%$, thereby

175 mathematically constraining the (co)variation in the pheromone and biasing the analysis. We

176 therefore transformed pheromone measurements to log-contrasts for all down-stream analyses in

177 this study. This approach breaks the interdependency and normalizes the data. Since the divisor

178 used in the contrast of the variable can no longer be part of any downstream analyses, we chose

179 14:Ald as the divisor, because this component has a small but clearly detectable peak in the

180 chromatogram, while it is irrelevant for male response behavior (Heath et al., 1990). Prior to

181 downstream analyses, samples with a $\chi^{2}$-distributed Mahalanobis distance score (calculated using

182 the 'mahalanobis' function in the 'stats' package) that exceeded a threshold value corresponding

183 to a Bonferroni-corrected $\mathrm{P}$-value < 0.05 were removed, which resulted in removing 64 out of a

184 total of 2,861 samples. These samples showed abnormal pheromone ratios and were present

185 across all selection and control lines and generations. We are therefore confident that these 
samples represent outliers, e.g. due to extraction or measurement errors, and are not representative of any relevant biological phenomena.

\section{Selection}

Since phenotyping consisted of extracting the sex pheromone gland invasively, selection was

190 performed post-mating. Each generation, we continued with those families that had a maternal

191 phenotype satisfying an increasingly stringent threshold. Initially, a so-called "high" and "low"

192 line were started with offspring from females with a relative amount of acetates above $22 \%$ or

193 below 16\%, respectively. These values were based on the distribution of the relative amounts of

194 acetates in the starting population, representing the 1st and 3rd quantile. These thresholds were

195 kept for the first 3 generations. In subsequent generations, we increased these thresholds to $>24 \%$

196 or $<14 \%$ (generation $4-5$ ), and $>26 \%$ or $<12 \%$ (generation $6-9$ ). The high line consisted of a

197 total of 2,236 breeding females across nine generations, ranging from 189 - 295 matings per

198 generation. After outlier removal, a total of 1,234 high line females were phenotyped, or between

19989 and 171 per generation during the selection phase. The low line consisted of 2,250 breeding

200 females (189 - 296 per generation) and, after outlier removal, phenotypes were measured for a

201 total of 1,180 females (57 - 169 per generation). We stopped selecting in generation 10, but

202 continued to phenotype $\sim 25$ females per line and per generation until generation 13 . Throughout

203 the selection experiment, we also maintained a control line from which we phenotyped between

2046 and 111 females (median 20 females) per generation. For detailed sample sizes see Table S1.

\section{Phenotypic selection response}

206 To test the hypothesis that acetates can respond to selection and that other pheromone

207 components change due to indirect selection, the selection responses as measured by the log-

208 contrasts were visualized using the R-package 'ggplot'. In addition, we also calculated the

209 average difference between selected and control lines in units of standard deviation of the

210 starting population. 
213 To test whether genetic variances decreased with selection and whether the structure of the

214 genetic variance-covariance matrix was affected by selection, we first ran multi-response animal

215 models implemented in the R-package 'MCMCglmm'. We limited our analyses to the four

216 components that have been show to affect male response: Z9-16:Ald, Z11-16:Ald, Z11-16:OAc,

217 and Z11-16:OH (in all cases, the log-contrast to 14:Ald was used). Model formulation roughly

218 followed the MCMCglmm manual (Hadfield, 2010) and Jarrod Hadfield's course notes

219 (Hadfield, 2012), as well as the animal model tutorial by Pierre de Villemereuil (Villemereuil,

220 2012). High and low line individuals had separate pedigrees, but shared ancestors for which we

221 had pedigree data up to three generations prior to the onset of the experiment (in total 1,065

222 breeding females). To minimize the influence from priors on the covariances, for which we had

223 no prior expectations with high degrees of belief, we formulated flat, uninformative priors for the

224 variance-covariance structure of the random effect ('animal'). To evaluate the models, we

225 checked chain convergence and assessed effective sample sizes and levels of autocorrelation. To

226 check whether the prior did not contribute unduly to posterior estimates, we also compared

227 genetic (co)variance estimates among univariate, bi-variate, and full (tetra-variate) models and

228 using different, more informative priors. We found models with flat priors to perform best.

229 To observe changes in genetic (co)variances at higher sample sizes, we partitioned the data in the

230 starting generation (generation 0$)$ and generation blocks of three generations each $(1-3,4-6,7$

$231-9)$ for both the high and low line. For each generation block, we obtained posterior estimates of

232 the additive genetic variance, $\mathrm{V}_{\mathrm{A}}$ for each of the four log-contrasts from the multi-response

233 animal model. For each posterior sample of $\mathrm{V}_{\mathrm{A}}$, we calculated the coefficient of additive genetic

234 variance, $\mathrm{CV}_{\mathrm{A}}$ (where $C V_{A}=\frac{V_{A}}{\text { phenotypic mean }}$ ), which provides a standardized measure of the

235 evolvability of a trait that is independent of other variance components. We also extracted the

236 posterior genetic correlations and visually inspected pairwise genetic correlations between Z11-

237 16:OAc and each of the other three components. Lastly, we examined changes in the $\mathrm{G}$ matrix by

238 inspecting the trait loadings on genetic principle components. The four genetic principle

239 components were obtained for every posterior sample using the 'eigen' function. We focused on

240 the first two axes, gPC1 (also known as $g_{\max }$, the direction in phenotypic space containing the

241 largest fraction of the genetic variance) and gPC2, because these axes jointly described $>90 \%$ of

242 the genetic variance (see Results). 


\section{RESULTS}

\section{Selection response in sex pheromone}

245 All three acetates responded to selection for higher/lower relative total amounts of acetates in the 246 high and low line, respectively (Fig 2). The response was more pronounced in the more abundant 247 Z9 and Z11 isomers compared to the less abundant Z7-16:OAc (Fig 2). The selection response 248 was characterized by an immediate response in the direction of selection (from generation 0 to generation 1), followed by a gradual progression towards increasing/decreasing relative amounts in the respective selection lines. In the low line, the selection response flattened after cessation of selection, while in the high line, the average ratio of Z11:16:OAc to 14:Ald was higher in

252 generations 11-13 compared to generations 7-10 (Fig 2D).
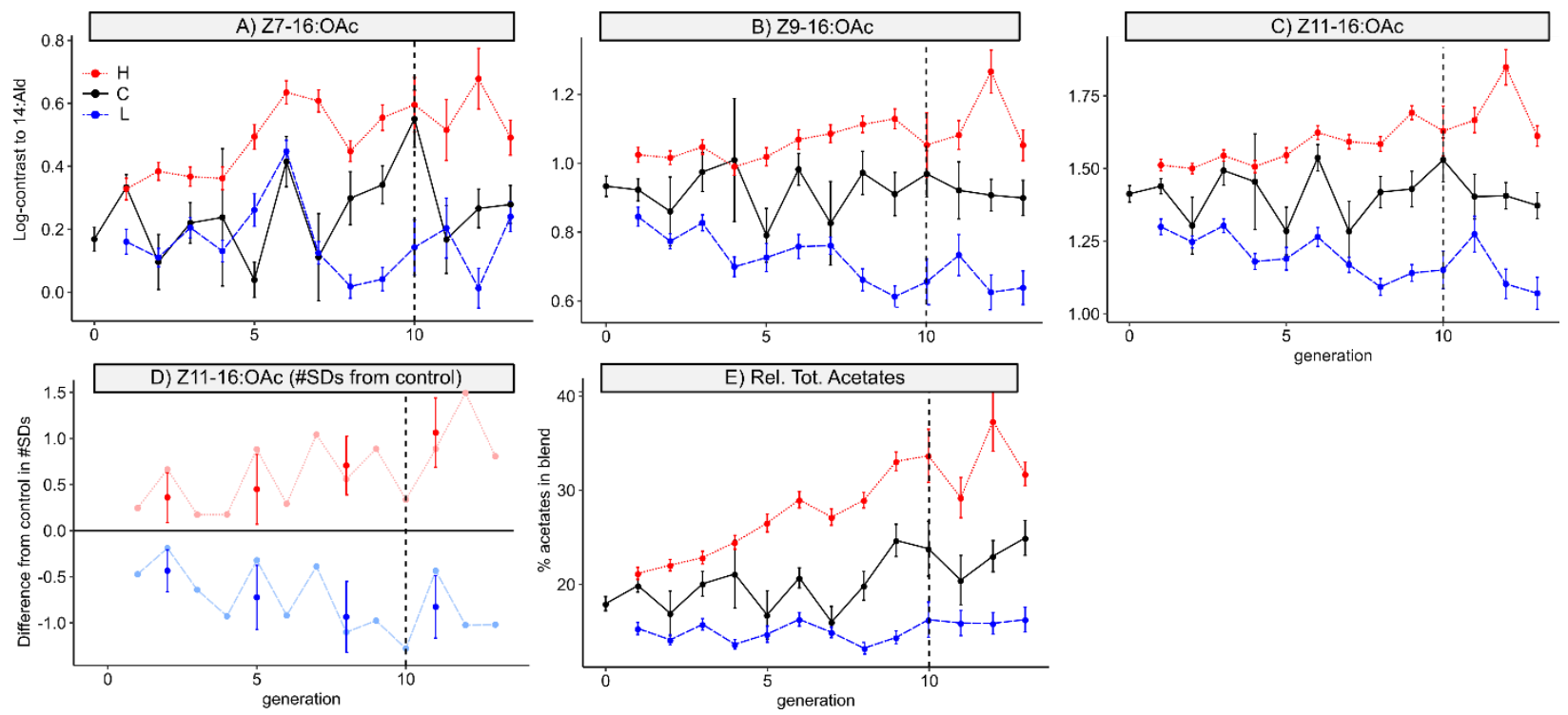

254 Fig 2. Selection response in the acetates. Values shown are mean \pm SEM. The control line is indicated in black, the high line in red (dotted) and the low line in blue (dashed). The dashed vertical line indicates the generation with adult females that developed from the last offspring that went through selection. A - C: Selection responses of the three acetates log-contrasted to 14:Ald. For the control line, 111 females were phenotyped in generation zero, 71 in generation one, and between 6 and 36 for the remaining generations. For the low and high line, respectively $189-$ 296 and 189 - 295 females per generation were phenotyped. D: Difference in phenotypic means between selection lines and control in units of starting population standard deviation. The points and error bars with brighter hues show the average \pm standard deviation for generation $1-3,4-6,7-10$, and $11-13$, respectively. E: Selection response of the relative total amount of acetates. 
The other pheromone components showed no differentiation among selection lines or between selected and control lines (Fig 3A-C). The total amount of pheromone measured across the 11 biologically active components remained constant through time, indicating that changing ratios were independent of the pheromone titer (Fig 3D).
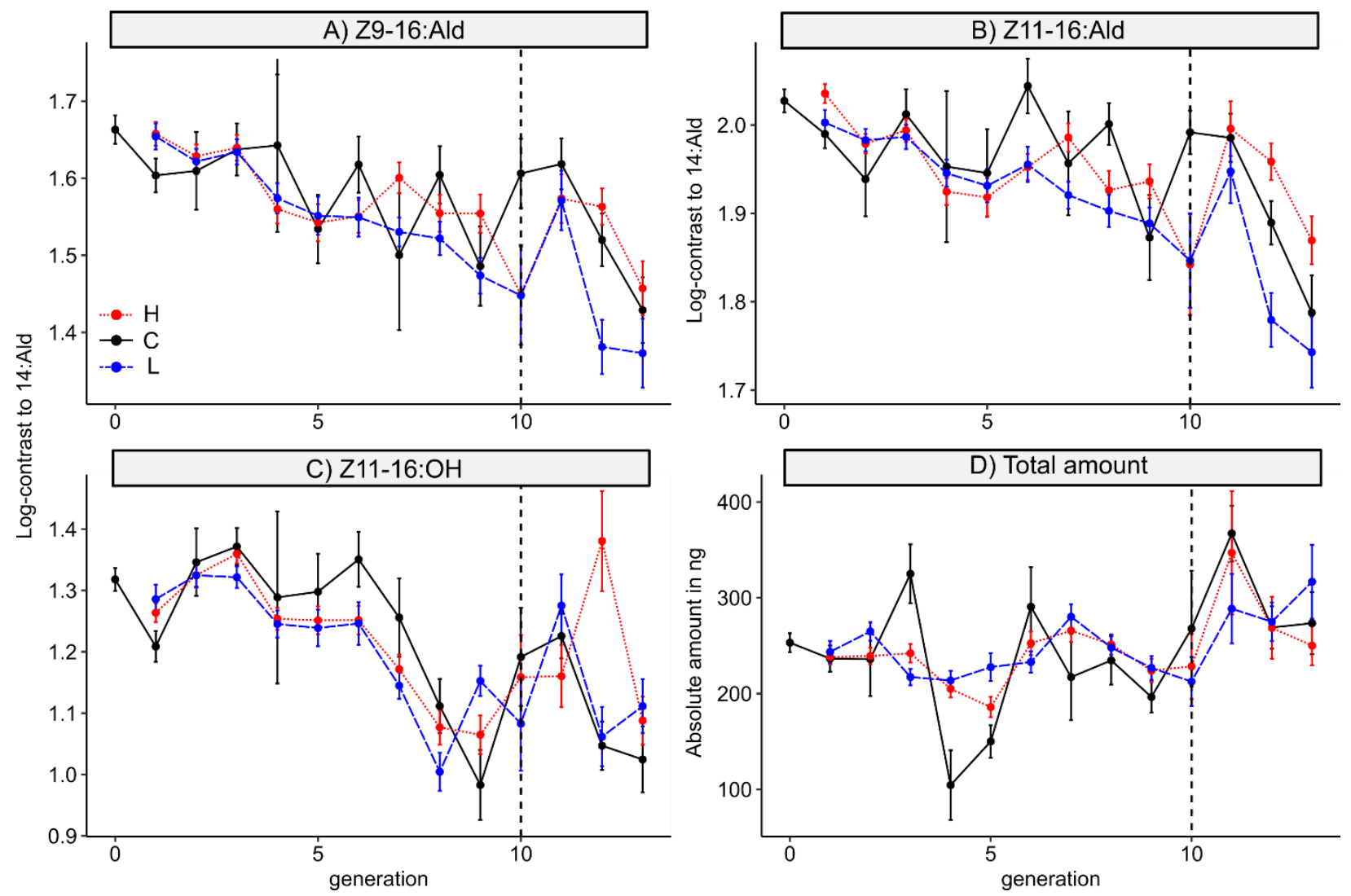

Fig 3. Indirect selection response. Values shown are mean \pm SEM for log-contrasts to 14:Ald of the three pheromone components that make up the minimal blend for male attraction and for the absolute amount of pheromone across all 11 biologically active components. The control line is indicated in black, the high line in red and the low line in blue. Dashed vertical lines indicate the generation with adult females that developed from the last offspring that went through truncation selection. Sample sizes as in Fig 2.

\section{Selection response in genetic (co)variances}

274 In testing whether the selection response was associated with (i) a reduction in genetic variance 275 in Z11-16:OAc (a change in the diagonal elements of the G matrix) and (ii) re-orientation of 276 genetic covariances among pheromone components (changes in the off-diagonal elements of the 277 G matrix), we found no evidence for decreasing genetic variance in Z11-16:OAc (Fig 4A) or in the other components (Fig S1). In contrast to genetic variances, we did find variation in the 
genetic covariance structure across generations. First, the posterior mode of the pairwise genetic

280 correlations differed between the starting and selected generations. Although the change in the

281 covariance between Z11-16:OAc and the two aldehydes was limited (Fig 4B,C), genetic

282 covariance between Z11-16:OAc and Z11-16:OH switched direction (from negative to positive)

283 and became more pronounced, especially in the low line (Fig 4D).
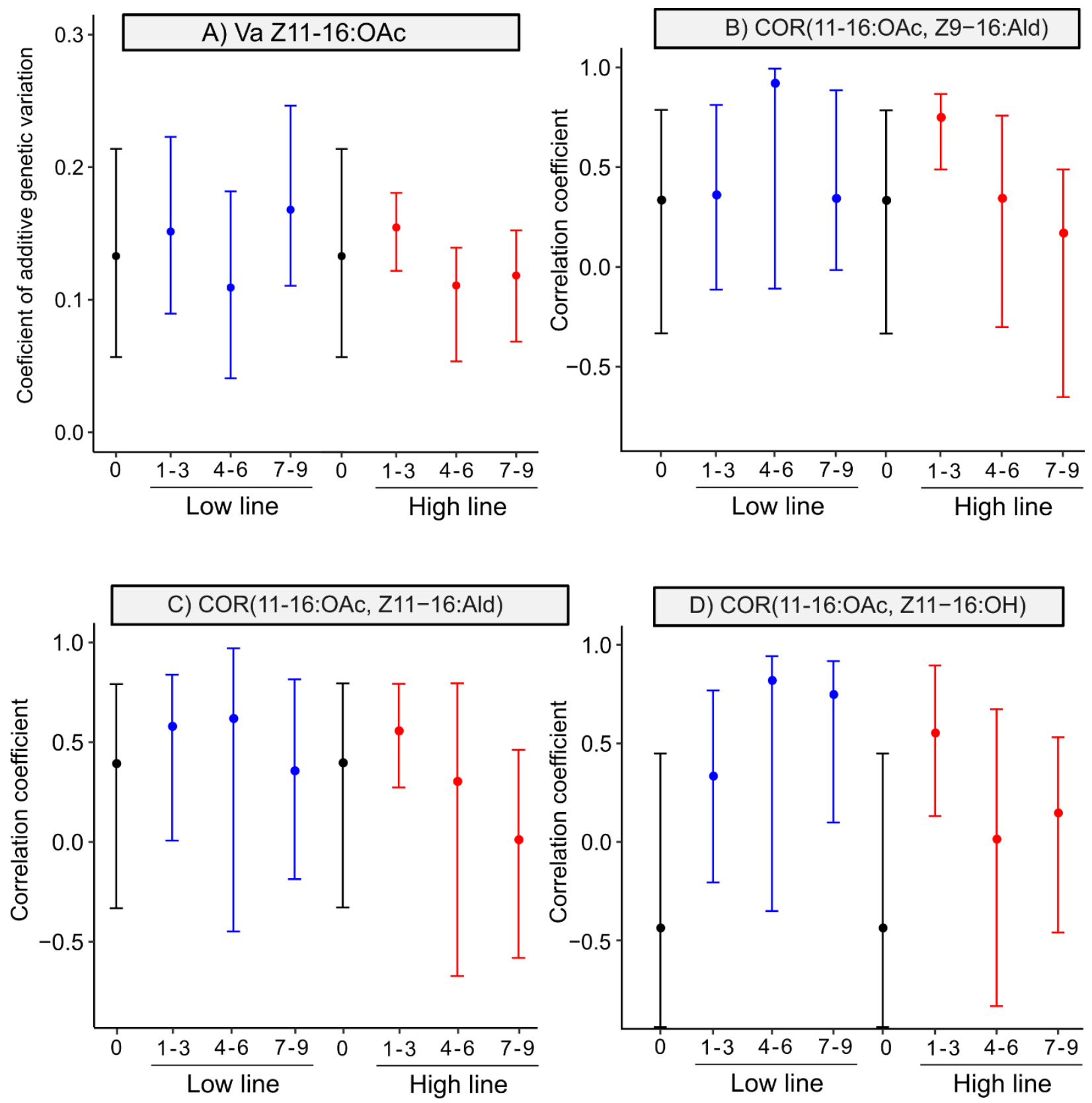
Fig 4. Genetic variation and correlations. The posterior mode and 95\% HPD interval of the genetic correlation between the log-contrast of Z11-16:OAc on 14:Ald and each of the other three log-contrasts is shown for the starting generation ( $\mathrm{S}$, in black), the low (blue) and high (red) lines. 111 females were phenotyped for the starting generation. For the low line, 443, 284, and 330 phenotyped females were in generation blocks 1-3, 4-6, and 7-9, respectively. For the high line 465, 324, and 344 phenotyped females were in generation block 1-3, 4-6, and 7-9, respectively. Pedigree data were obtained for 1,065 mating pairs prior to the first generation of selection and for an additional 2,250 and 2,236 pairs for the low and high line, respectively.

Second, the partitioning of genetic variance among gPC1 and gPC2 evolved. The first two genetic principle components changed in magnitude of their eigenvalue (i.e. the amount of genetic variance they describe) and in their loadings (i.e. in their correlation with the pheromone components; Fig 5). More of the total genetic variance across the four components was in the direction of gPC1 in the high and low lines ( 80\%) compared to the starting generation (59.1\%). This means that more of the genetic variance of the sex pheromone was biased towards a single dimensions in phenotypic space. Moreover, variation in the component under selection, Z1116:OAc, was more strongly associated with gPC2 and less with gPC1 in generations after compared to before selection, while the other traits showed a trend in the opposite direction (Fig 5). These changes in the G matrix resulted in a more modular pheromone blend, because Z1116:OAc, which was under selection here, became uncoupled from the components that were not under selection.

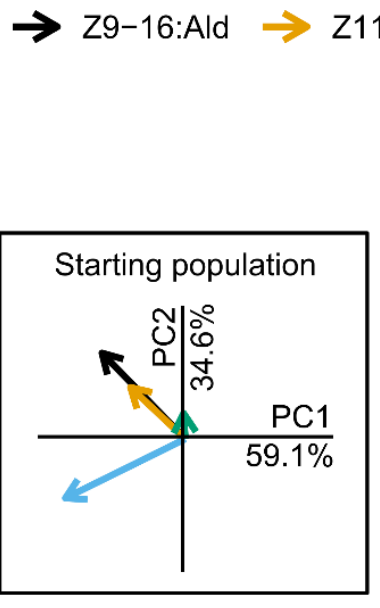

305

306
Z11-16:Ald $\rightarrow$ Z11-16:OAc $\rightarrow$ Z11-16:OH
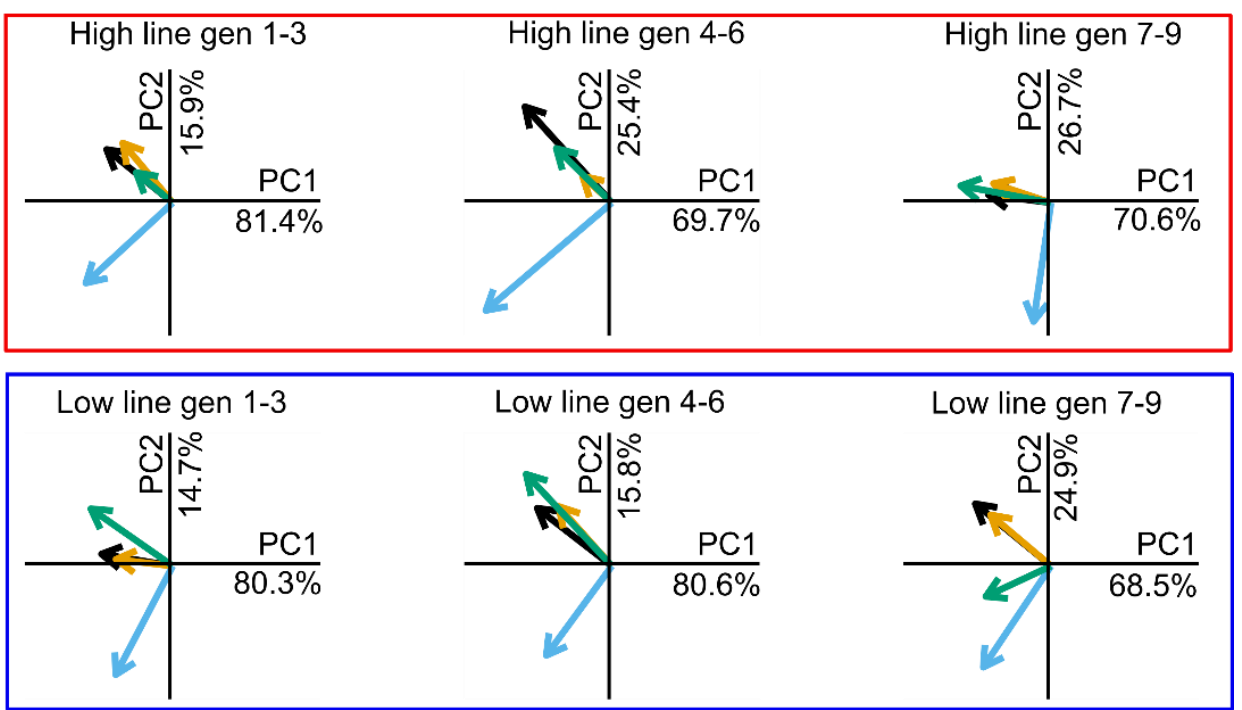

Low line gen 7-9

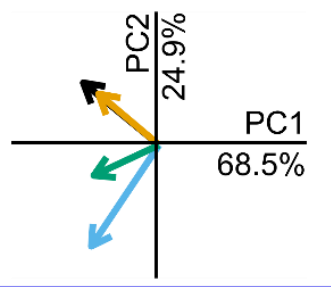

Fig 5. Eigen-analysis of genetic principle components. For the starting generation and generation 1-3, 4-6, and 7-9 of the low (top) and high (bottom) lines, the posterior mode of the correlation between each of the pheromone ratios 
and the first and second principle components is shown by the direction and size of the arrows. The genetic variance explained by $\mathrm{PC} 1$ and $\mathrm{PC} 2$ is indicated on the axes.

\section{DISCUSSION}

312 In this study, we investigated how the selection response depends on and shapes the genetic

313 architecture of a multicomponent sex pheromone signal. Through truncation selection, we

314 gradually increased and decreased the relative amount of acetates across 10 generations. As we

315 hypothesized, we found that the acetates responded readily to selection, diverging three-quarters

316 of a standard deviation away from the control line in 10 generations in both the high and low

317 lines. However, in contrast to our hypothesis that selection would also result in indirect responses

318 in the other traits, we found that the response was limited to the acetates only. In addition, we

319 found that levels of genetic variance were constant through time, opposite to the expected

320 erosion of genetic variance in response to selection. Lastly, in line with our expectations, the

321 genetic covariance structure changed in a way that likely facilitated a selection response in the

322 pheromone. We discuss each of these results further below.

\section{Univariate responses in multicomponent signals}

324 In nature, correlational selection may be quite common (Svensson et al., 2021) and sexual

325 signals are often found to be subject to multivariate stabilizing and directional selection (Bentsen

326 et al., 2006; Blankers et al., 2015; Brooks et al., 2005; Devigili et al., 2015; Fisher et al., 2009;

327 Gerhardt and Brooks, 2009; Hine et al., 2011; Oh and Shaw, 2013; Ryan and Rand, 2003;

328 Tanner et al., 2017). In our experiment, we applied a univariate selection gradient on the relative 329 amount of Z11-16:OAc to consider the evolvability of multivariate traits to univariate selection

330 gradients. We justified this univariate selection based on the found geographic variation in the

331 relative amount of Z11:16OAc in $H$. subflexa. Females are likely specifically selected for higher

332 rates of acetates to avoid heterospecific mate attraction in regions where the congener $H$.

333 virescens occurs (Groot et al., 2009a).

334 Our observed selection response shows that there are no phenotypic or genetic constraints to the 335 evolution of Z11-16:OAc (or the other acetates), as we reached similar or even higher levels of 336 divergence compared to those observed across the range of $H$. subflexa. After 10 generations in 
our selection experiment, the high line individuals had on average almost $20 \%$ more Z1116:OAc compared to the low line (Fig 2E), while H. subflexa populations in the eastern US were found to have blends with up to $10 \%$ more Z11-16:OAc compared to populations in the southwestern US and Mexico (Groot et al., 2009a). The selection response that we found was also surprisingly univariate, as only the three acetate esters and none of the other components showed divergence between the high and low lines. This indicates that the acetates can evolve independently from the other components. This is surprising, both in the context of the observed genetic correlations in the starting population and in later generations (Fig 4) and in the context of what is known about the shared biochemical pathways across the pheromone components (Groot et al. 2009a; Fig 1). However, it is not an uncommon result of artificial selection experiments (Hill and Caballero, 1992; Saltz et al., 2017), including for sexual signals (Ritchie and Kyriacou, 1996). Thus, genetic correlations among sexual signal components may quickly dissolve in the absence of correlational selection, which likely facilitates their evolution on short time scales in response to directional selection.

351 We acknowledge that our study does not include replicate lines in the experiment. This is 352 because each selection line consisted of hundreds of single pair matings per generation, as $H$. 353 subflexa is highly sensitive to inbreeding. We thus needed to maintain large populations of 354 individuals that are mated in pairs and also individually reared to avoid cannibalism, which makes these experiments very laborious. Therefore, we chose to rear two lines in separate

356 directions rather than two replicates of only the high or low line. The fact that we found parallel

357 results between the lines in the selection response both in phenotypic means and genetic

358 covariances lends confidence to the patterns observed. However, we do avoid drawing

359 conclusions based on differences between the lines, because these can both reflect differential 360 selection effects or sampling variance.

\section{No change in genetic variance}

362 The effect of selection on genetic variation is a key topic in evolutionary biology. Strong 363 stabilizing or directional selection is expected to erode genetic variance (Barton and Turelli, 364 1989; Estes and Arnold, 2007). However, when many loci are contributing to variation in the 365 trait under selection, selection might leave no detectable signature on the genetic variance 366 associated with that trait (Bulmer, 1971; Johnson and Barton, 2005). Although our truncated 
selection increased and decreased the phenotypic mean by three-quarters of a standard deviation in the high and low line, respectively, we observed no significant decrease in the coefficient of additive genetic variance. There are several explanations for this observation. First, several genetic factors may be involved. Previously, genetic mapping studies revealed several QTL underlying difference in sex pheromone composition in H. subflexa (Groot et al., 2013). Since variation in pheromone composition in $H$. subflexa is also explained by fitness variation (Blankers et al., 2021), many different genetic factors likely contribute small additional fractions to the total variance. Another explanation comes from population genetics: re-orientation of genetic covariances in response to bottlenecks may free up additive genetic variance, thereby paradoxically increasing levels of genetic variance (Carson, 1990; Templeton, 2008). Since truncation selection is effectively a non-random bottleneck of the population, this may also explain the maintenance of genetic variance that we observed. Lastly, to avoid inbreeding depression, we maintained large populations and avoided mating first and second-degree relatives. Such a mating scheme likely counteracts some loss in genetic variability due to selection (Du et al., 2021).

\section{Changing genetic covariance}

384 Specifically, the correlation between Z11-16:OH and Z11-16:OAc changed in both sign and 385 magnitude. Since both Z11-16:OH and Z11-16:OAc reduce heterospecific mate attraction 386 (Vetter and Baker, 1983; Vickers and Baker, 1997), and both traits covary with fitness and are 387 condition-dependent (Blankers et al., 2021), the negative genetic correlation in the starting 388 population could be the result of a trade-off that is selected against when artificial selection favors higher rates of acetates.

390 Tracking changes in G matrices through time, we observed another interesting phenomenon:

391 after selection, the genetic variance in Z11-16:OAc was more strongly associated with genetic

392 (g) PC2 compared to before selection, while genetic variance described by gPC1 increased

393 relative to the other gPCs. Thus, more of the genetic variance was allocated in fewer dimensions

394 of genetic variation, while the component under selection was uncoupled from the major

395 dimension of genetic variance. We acknowledge that the observed changes are subtle and that 396 they cannot be unequivocally attributed to selection. However, we observed similar patterns of 
397 G matrix evolution in the high and low line and these patterns fit the prediction that genetic

398 variances and covariances are reoriented to align the phenotype with the dominant direction of

399 selection (Melo and Marroig, 2014). These changes in the G matrix can likely facilitate a more

400 specific response where only Z11-16:OAc evolves in the absence of indirect selection responses:

401 relative to the selection regime, the modularity (i.e. the genetic independence of trait modules) of

402 the pheromone increased.

\section{Conclusion}

404 From our results, we conclude that: 1) pheromone components in H. subflexa can evolve in 405 response to selection independent of other components of the sex pheromone; 2) selection need 406 not erode the genetic variance in order to drive this phenotypic change; 3) selection alters the 407 genetic correlations among pheromone components. Our study thus shows that univariate 408 selection responses in multicomponent sexual signals are possible, even though pairwise genetic 409 correlations were high both prior to and during selection. Our study also shows that sexual signal 410 components can respond to selection without reductions in genetic variance, but with rapid 411 changes in the genetic covariance structure. These results correspond to geographic variation in 412 the sexual signal found in this species and help to explain how sexual signals in general can 413 respond to selection without running into constraints from indirect selection responses or 414 depleted genetic variation. This may be one reason why sexual signals have evolved rapidly and 415 repeatedly throughout the evolutionary history of animal taxa, thereby contributing to the 416 speciation process.

\section{ACKNOWLEDGEMENTS}

418 We thank Dennis van Veldhuizen for his invaluable contribution to the rearing of our moths. We 419 also thank Laura Caton, Frederica Lotito, and Lilian Seip for their help with the selection lines.

420 We further thank Jet ten Berge for digitizing the pedigree, Michiel van Wijk for commenting on 421 a previous version of the manuscript, and all members of the Groot lab for their helpful and 422 insightful discussions.

\section{COMPETING INTERESTS}

424 The authors declare no conflict of interest 


\section{AUTHOR CONTRIBUTIONS}

426 All authors contributed to the conceptualization of the study; TB, EF, and EBS performed the

427 experiments; TB analyzed the data; and all authors contributed to writing the manuscript.

\section{FUNDING}

429 This study was funded by the following grants: the European Union's Horizon 2020 research and 430 innovation programme under the Marie Skłodowska-Curie grant agreement no. 794254 awarded

431 to TB, NWO grant award ALWOP.2015.075 awarded to ATG, an IMPRS grant of the Max

432 Planck Society awarded to EF, and an ASAB Research grant awarded to EBS.

\section{DATA ACCESSIBILITY}

434 Data and analysis scripts will be archived in Dryad.

\section{FIGURE LEGENDS}

436 Figure 1. Simplified biosynthetic pathway of the Heliothis subflexa sex pheromone, modified

437 from (Groot et al., 2009b) with permission. Desaturation and $\beta$-oxidation produce mono-

438 unsaturated acyl-CoA precursors from 18 or 16 carbon acyl CoA derivatives, which are then

439 modified to form acetate esters, alcohols, and aldehydes through specific enzymatic conversions.

440 The $\Delta 9$ and $\Delta 11$ desaturases create a double bond between the $9^{\text {th }}$ and $10^{\text {th }}$ or $11^{\text {th }}$ and $12^{\text {th }}$ carbon

441 of the 18- or 16-carbon acyl-CoA derivatives, respectively. $\beta$-oxidation shortens the chain length

442 from 18 to 16 carbons. Note that (Z)9-16 acyl-CoA can be formed through two alternative

443 routes. The compounds present in the pheromone glands of $H$. subflexa are in grey boxes.

445 Fig 2. Selection response in the acetates. Values shown are mean \pm SEM. The control line is 446 indicated in black, the high line in red (dotted) and the low line in blue (dashed). The dashed 447 vertical line indicates the generation with adult females that developed from the last offspring 448 that went through selection. A - C: Selection responses of the three acetates log-contrasted to 449 14:Ald. For the control line, 111 females were phenotyped in generation zero, 71 in generation 450 one, and between 6 and 36 for the remaining generations. For the low and high line, respectively $451189-296$ and 189 - 295 females per generation were phenotyped. D: Difference in phenotypic 452 means between selection lines and control in units of starting population standard deviation. The 
453 points and error bars with brighter hues show the average \pm standard deviation for generation $1-$ $4543,4-6,7-10$, and $11-13$, respectively. E: Selection response of the relative total amount of 455 acetates.

Fig 3. Indirect selection response. Values shown are mean \pm SEM for log-contrasts to 14:Ald of the three pheromone components that make up the minimal blend for male attraction and for the absolute amount of pheromone across all 11 biologically active components. The control line is indicated in black, the high line in red and the low line in blue. Dashed vertical lines indicate the generation with adult females that developed from the last offspring that went through truncation selection. Sample sizes as in Fig 2.

Fig 4. Genetic variation and correlations. The posterior mode and 95\% HPD interval of the genetic correlation between the log-contrast of Z11-16:OAc on 14:Ald and each of the other three log-contrasts is shown for the starting generation ( $\mathrm{S}$, in black), the low (blue) and high (red) lines. 111 females were phenotyped for the starting generation. For the low line, 443, 284, and 330 phenotyped females were in generation blocks 1-3, 4-6, and 7-9, respectively. For the high line 465, 324, and 344 phenotyped females were in generation block 1-3, 4-6, and 7-9, respectively. Pedigree data were obtained for 1,065 mating pairs prior to the first generation of selection and for an additional 2,250 and 2,236 pairs for the low and high line, respectively.

Fig 5. Eigen-analysis of genetic principle components. For the starting generation and generation 474 1-3, 4-6, and 7-9 of the low (top) and high (bottom) lines, the posterior mode of the correlation 475 between each of the pheromone ratios and the first and second principle components is shown by 476 the direction and size of the arrows. The genetic variance explained by PC1 and PC2 is indicated 477 on the axes.

480 Allison, J. D. and Cardé, R. T. (2008). Male pheromone blend preference function measured in 
choice and no-choice wind tunnel trials with almond moths, Cadra cautella. Anim. Behav. 75, 259-266.

Andersson, M. (1994). Sexual Selection. Princeton University Press.

Armbruster, W. S., Pélabon, C., Bolstad, G. H. and Hansen, T. F. (2014). Integrated phenotypes: understanding trait covariation in plants and animals. Philos. Trans. R. Soc. B Biol. Sci. 369, 20130245.

Arnold, S. J., Bürger, R., Hohenlohe, P. A., Ajie, B. C. and Jones, A. G. (2008). Understanding the evolution and stability of the G-matrix. Evolution 62, 2451-2461.

Barton, N. and Turelli, M. (1989). Evolutionary Quantitative Genetics: How Little Do We Know. Annu. Rev. Genet. 23, 337-370.

Bégin, M. and Roff, D. A. (2003). The constancy of the G matrix through species divergence and the effects of quantitative genetic constraints on phenotypic evolution: a case study in crickets. Evolution 57, 1107-1120.

Bentsen, C. L., Hunt, J., Jennions, M. D. and Brooks, R. (2006). Complex multivariate sexual selection on male acoustic signaling in a wild population of Teleogryllus commodus. Am. Nat. 167, E102-E116.

Berner, D., Stutz, W. E. and Bolnick, D. I. (2010). Foraging trait (co)variances in stickleback evolve deterministically and do not predict trajectories of adaptive diversification. Evolution

Blankers, T., Gray, D. A. and Hennig, R. M. (2017). Multivariate Phenotypic Evolution: (N. Y). 64, 2265-2277.

Blankers, T., Hennig, R. M. and Gray, D. A. (2015). Conservation of multivariate female preference functions and preference mechanisms in three species of trilling field crickets. $J$. Evol. Biol. 28, 630-641. 
210180.

Brooks, R., Hunt, J., Blows, M. W., Smith, M. J., Bussière, L. F. and Jennions, M. D. (2005). Experimental Evidence for Multivariate Stabilizing Selection. Evolution (N. Y). 59, 871-880.

Bulmer, M. G. (1971). The Effect of Selection on Genetic Variability. Am. Nat. 105, 201-211.

Candolin, U. (2003). The use of multiple cues in mate choice. Biol. Rev. 78, 575-595.

Cardé, R. T. and Haynes, K. F. (2004). Structure of the pheromone communication channel in moths. In Advances in insect chemical ecology (ed. Cardé, R. T.) and Millar, J. G.), pp. 283-332. Cambridge University Press Cambridge.

Careau, V., Wolak, M. E., Carter, P. A. and Garland, T. (2015). Evolution of the additive genetic variance-covariance matrix under continuous directional selection on a complex behavioural phenotype. Proc. R. Soc. B Biol. Sci. 282,.

Carson, H. L. (1990). Increased genetic variance after a population bottleneck. Trends Ecol. Evol. 5, 228-230.

Chenoweth, S. F. and Blows, M. W. (2006). Dissecting the complex genetic basis of mate choice. Nat. Rev. Genet. 7, 681-692.

Cheverud, J. M. (1996). Developmental Integration and the Evolution of Pleiotropy. Integr. Comp. Biol. 36, 44-50.

Cole, G. L. and Endler, J. A. (2015). Variable environmental effects on a multicomponent sexually selected trait. Am. Nat. 185, 452-468.

Coyne, J. A. and Orr, A. H. (2004). Speciation. Sinauer Associates, Sunderland, MA.

Devigili, A., Evans, J. P., Di Nisio, A. and Pilastro, A. (2015). Multivariate selection drives concordant patterns of pre- and postcopulatory sexual selection in a livebearing fish. Nat. Commun. 6, 8291.

Du, M., Bernstein, R., Hoppe, A. and Bienefeld, K. (2021). Short-term effects of controlled mating and selection on the genetic variance of honeybee populations. Heredity (Edinb). 733-747. 
Eroukhmanoff, F. (2009). Just how much is the G-matrix actually constraining adaptation? Evol. Biol. 36, 323-326.

Estes, S. and Arnold, S. J. (2007). Resolving the paradox of stasis: models with stabilizing selection explain evolutionary divergence on all timescales. Am. Nat. 169, 227-244.

Ferveur, J. F. (2005). Cuticular hydrocarbons: Their evolution and roles in Drosophila pheromonal communication. Behav. Genet. 35, 279-295.

Fisher, H. S., Mascuch, S. J. and Rosenthal, G. G. (2009). Multivariate male traits misalign with multivariate female preferences in the swordtail fish, Xiphophorus birchmanni. Anim. Behav. 78, 265-269.

Garant, D., Hadfield, J. D., Kruuk, L. E. B. and Sheldon, B. C. (2008). Stability of genetic variance and covariance for reproductive characters in the face of climate change in a wild bird population. Mol. Ecol. 17, 179-188.

Gerhardt, H. C. and Brooks, R. (2009). Experimental analysis of multivariate female choice in gray treefrogs (Hyla versicolor): Evidence for directional and stabilizing selection. Evolution (N. Y). 63, 2504-2512.

Gosden, T. P. and Chenoweth, S. F. (2014). The evolutionary stability of cross-sex, cross-trait genetic covariances. Evolution (N. Y). 68, 1687-1697.

Grether, G. F., Kolluru, G. R. and Nersissian, K. (2004). Individual colour patches as multicomponent signals. Biol. Rev. Camb. Philos. Soc. 79, 583-610.

Groot, A. T., Fan, Y., Brownie, C., Jurenka, R. A., Gould, F. and Schal, C. (2005). Effect of PBAN on pheromone production by mated Heliothis virescens and Heliothis subflexa females. J. Chem. Ecol. 31, 15-28.

Groot, A. T., Horovitz, J. L., Hamilton, J., Santangelo, R. G., Schal, C. and Gould, F. (2006). Experimental evidence for interspecific directional selection on moth pheromone communication. Proc. Natl. Acad. Sci. U. S. A. 103, 5858-5863.

Groot, A. T., Santangelo, R. G., Ricci, E., Brownie, C., Gould, F. and Schal, C. (2007). Differential Attraction of Heliothis subflexa Males to Synthetic Pheromone Lures in Eastern 
US and Western Mexico. J. Chem. Ecol. 33, 353-368.

Groot, A. T., Inglis, O., Bowdridge, S., Santangelo, R. G., Blanco, C., López, J. D., Vargas,

$$
\text { A. T., Gould, F. and Schal, C. (2009a). Geographic and temporal variation in moth }
$$
chemical communication. Evolution (N. Y). 63, 1987-2003.

Groot, A. T., Estock, M. L., Horovitz, J. L., Hamilton, J., Santangelo, R. G., Schal, C. and

$$
\text { Gould, F. (2009b). QTL analysis of sex pheromone blend differences between two closely }
$$

Groot, A. T., Blanco, C. A., Claßen, A., Inglis, O., Santangelo, R. G., Lopez, J., Heckel, D. related moths: insights into divergence in biosynthetic pathways. Insect Biochem. Mol. Biol.

Groot, A. T., Staudacher, H., Barthel, A., Inglis, O., Schöfl, G., Santangelo, R. G., Gebauerintra- and interspecific variation in a sex pheromone. Mol. Ecol. 22, 1065-1080.

Hadfield, J. D. (2010). MCMC Methods for Multi-Response Generalized Linear Mixed Models: The MCMCglmm R Package. J. Stat. Softw. 33, 1-22.

Hadfield, J. D. (2012). MCMCglmm Course Notes.

Heath, R. R., Mitchell, E. R. and Cibrian Tovar, J. (1990). Effect of release rate and ratio of

Higham, J. P. and Hebets, E. A. (2013). An introduction to multimodal communication. Behav. Ecol. Sociobiol. 67, 1381-1388.

Hill, W. G. and Caballero, A. (1992). Artificial selection experiments. Annu. Rev. Ecol. Syst. 23, 287-310.

Hine, E., McGuigan, K. and Blows, M. W. (2011). Natural selection stops the evolution of male attractiveness. Proc. Natl. Acad. Sci. 108, 3659-64.

588 Johnson, T. and Barton, N. (2005). Theoretical models of selection and mutation on 
quantitative traits. Philos. Trans. R. Soc. B Biol. Sci. 360, 1411-1425.

Jones, A. G., Arnold, S. J. and Bürger, R. (2003). Stability of the g-matrix in a population experiencing pleiotropic mutation, stabilizing selection, and genetic drift. Evolution (N. Y). 57, 1747-1760.

Kárpáti, Z., Tasin, M., Cardé, R. T. and Dekker, T. (2013). Early quality assessment lessens pheromone specificity in a moth. Proc. Natl. Acad. Sci. 110, 7377 LP - 7382.

Lande, R. (1979). Quantitative genetic analysis of multivariate evolution, applied to brain: body size allometry. Evolution 33, 402-416.

Lande, R. and Arnold, S. J. (1983). The measurement of selection on correlated characters. Evolution (N. Y). 37, 1210-1226.

Linn, C. E. J., Campbell, M. G. and Roelofs, W. L. (1987). Pheromone components and active spaces: what do moths smell and where do they smell it? Science 237, 650-652.

Linn, C. E., Young, M. S., Gendle, M., Glover, T. J. and Roelofs, W. L. (1997). Sex pheromone blend discrimination in two races and hybrids of the European corn borer moth, Ostrinia nubilalis. Physiol. Entomol. 22, 212-223.

Lynch, M. and Walsh, B. (1998). Genetics and analysis of quantitative Traits. Sinauer, Sunderland, MA.

Melo, D. and Marroig, G. (2014). Directional selection can drive the evolution of modularity in complex traits. Proc. Natl. Acad. Sci. 112,

612 R Core Team (2019). R: A Language and Environment for Statistical Computing.

613 Revell, L. J. (2007). The G matrix under fluctuating correlational mutation and selection. Evolution (N. Y). 61, 1857-1872. 
Ritchie, M. G. (2007). Sexual Selection and Speciation. Annu. Rev. Ecol. Evol. Syst. 38, 79-102.

Ritchie, M. G. and Kyriacou, C. P. (1996). Artificial selection for a courtship signal in Drosophila melanogaster. Anim. Behav. 52, 603-611.

Roff, D. A. and Fairbairn, D. J. (2012). The evolution of trade-offs under directional and correlational selection. Evolution (N. Y). 66, 2461-2474.

Rowe, C. (1999). Receiver psychology and the evolution of multicomponent signals. Anim. Behav. 58, 921-931.

Ryan, M. J. and Rand, a S. (2003). Sexual selection in female perceptual space: how female túngara frogs perceive and respond to complex population variation in acoustic mating signals. Evolution 57, 2608-2618.

Saltz, J. B., Hessel, F. C. and Kelly, M. W. (2017). Trait Correlations in the Genomics Era. Trends Ecol. Evol. 32, 279-290.

Schaefer, H. M. and Ruxton, G. D. (2015). Signal Diversity, Sexual Selection, and Speciation. Annu. Rev. Ecol. Evol. Syst. 46, 573-592.

Schneider, D. (1992). 100 years of pheromone research - An essay on lepidoptera. Naturwissenschaften 79, 241-250.

Svensson, E. I., Arnold, S. J., Bürger, R., Csilléry, K., Draghi, J., Henshaw, J. M., Jones, A. the age of genomics. Nat. Ecol. Evol. 5,.

Tanner, J. C., Ward, J. L., Shaw, R. G. and Bee, M. A. (2017). Multivariate phenotypic

Templeton, A. R. (2008). The reality and importance of founder speciation in evolution. BioEssays 30, 470-479.

Uesugi, A., Connallon, T., Kessler, A. and Monro, K. (2017). Relaxation of herbivoremediated selection drives the evolution of genetic covariances between plant competitive and defense traits. Evolution (N. Y). 71, 1700-1709.

641 Vetter, R. S. and Baker, T. C. (1983). Behavioral responses of maleHeliothis virescens in a 
sustained-flight tunnel to combinations of seven compounds identified from female sex pheromone glands. J. Chem. Ecol. 9, 747-759.

Vickers, N. J. (2002). Defining a synthetic pheromone blend attractive to male Heliothis subflexa under wind tunnel conditions. J. Chem. Ecol. 28, 1255-1267.

Vickers, N. J. and Baker, T. C. (1997). Flight of Heliothis virescens males in the field in response to sex pheromone. Physiol. Entomol. 22, 277-285.

Villemereuil, P. de (2012). Animal Model Tutorial.

West-Eberhard, M. J. (2014). Darwin's forgotten idea: The social essence of sexual selection. Neurosci. Biobehav. Rev. 46, 501-508.

Wiens, J. J. and Tuschhoff, E. (2020). Songs versus colours versus horns: what explains the diversity of sexually selected traits? Biol. Rev. 95, 847-864.

Wilkins, M. R., Seddon, N. and Safran, R. J. (2013). Evolutionary divergence in acoustic signals : causes and consequences. Trends Ecol. Evol. 28, 156-166.

Wilkins, M. R., Shizuka, D., Joseph, M. B., Hubbard, J. K. and Safran, R. J. (2015). Multimodal signalling in the North American barn swallow: A phenotype network approach. Proc. R. Soc. B Biol. Sci. 282,.

Wolf, J. B., Leamy, L. J., Routman, E. J. and Cheverud, J. M. (2005). Epistatic pleiotropy and the genetic architecture of covariation within early and late-developing skull trait complexes in mice. Genetics 171, 683-694.

Zhu, J., Chastain, B. B., Spohn, B. G. and Haynes, K. F. (1997). Assortative mating in two pheromone strains of the cabbage looper moth, Trichoplusia ni. J. Insect Behav. 10, 805817. 